\title{
Center flux correlation in SU(2) Yang-Mills theory*
}

\author{
K. Langfeld, H. Reinhardt ${ }^{\dagger}$ and G. Schulze \\ Universität Tübingen, Germany \\ E-mail: kurt.langfeld@uni-tuebingen.de, h.reinhardt@uni-tuebingen.de
}

By using the method of center projection the center vortex part of the gauge field is isolated and its propagator is evaluated in the center Landau gauge, which minimizes the open 3-dimensional Dirac volumes of non-trivial center links bounded by the closed 2-dimensional center vortex surfaces. The center field propagator is found to dominate the gluon propagator (in Landau gauge) in the low momentum regime and to give rise to an OPE correction to the latter of $\sqrt{\sigma} / p^{3}$. The screening mass of the center vortex field vanishes above the critical temperature of the deconfinement phase transition, which naturally explains the second order nature of this transition consistently with the vortex picture. Finally, the ghost propagator of maximal center gauge is found to be infrared finite and thus shows no signal of confinement.

XXIIIrd International Symposium on Lattice Field Theory

25-30 July 2005

Trinity College, Dublin, Ireland

* supported in part by DFG Re 856/4-3

†peaker. 
Although confi nement has not yet been thoroughly understood, several pictures of confi nement have been developped, which received strong support by lattice calculations in recent years. Among these are the dual Meissner effect and the center vortex condensation (for a recent review see [1]).

A different confi nement mechanism was proposed by Gribov [2] and further elaborated by Zwanziger [3]. This mechanism is based on the infrared dominance of the fi eld confi gurations near the Gribov horizon, which gives rise to an infrared singular ghost propagator, which is considered to be a signal of confi nement [4]. In Landau gauge this infrared singularity (see fi g. 2) disappears, when center vortices are eliminated from the Yang-Mills ensemble [5]. Since the infrared singularities are caused by fi eld confi gurations on the Gribov horizon, one expects, that center vortices are on the Gribov horizon, which indeed can be shown to be the case [6]. The results of [5] and [6] show that, in Landau and Coulomb gauge, the center vortices are not only on the Gribov horizon, but they also dominate the infrared physics. This suggests, that the center vortices may be the confi ner in any gauge, which is very plausible since center vortices can, in principle, be defi ned in a gauge invariant way and after all confi nement is a gauge independent phenomenon.

In this talk I will further elaborate on the connection of the two confi nement scenarios described above, i.e. on the interplay between center vortices and ghosts. Using the method of center projection, we separate the confi ning center degrees of freedom from the remainig (non-confi ning) coset degrees of freedom and calculate the associate propagators as well as the corresponding ghost propagator. We will fi nd, that in contrast to the familiar Landau gauge, in the maximum center gauge the ghost propagator is infrared fi nite and thus shows no signal of confi nement. This result is not surprising since in this gauge the Faddeev-Popov operator does not feel the center part of the gauge fi eld. The confi ning center degrees of freedom constitute the center vortex fi eld. We calculate its propagator which is carefully extrapolated to the continuum limit. We will fi nd, that the center fi eld propagator dominates the infrared behaviour of the gluon propagator, while the ultraviolet behaviour of the latter is exclusively determined by the coset fi eld propagator. However, the center vortex fi eld gives rise to an correction to the gluon propagator of the form $\sqrt{\sigma} / p^{3}$ which indicates its relevance in the context of the operator product expansion.

Although the lattice provides a gauge invariant approach to Yang-Mills theory the transition to the continuum theory is fascilitated by using a gauge, in which the fi elds are smooth. The prototype of such a gauge is the wellknown Landau gauge

$$
\sum_{x, \mu} \operatorname{tr} U_{\mu}^{\Omega}(x) \stackrel{\Omega}{\longrightarrow} \max ,
$$

We will use various modifi cations of the Landau gauge to identify the center vortex content and the remaining coset part of the gauge fi eld as will be detailed further below.

To identify the center vortex content of a gauge fi eld, we use the method of center projection, which is based on the so-called maximal center gauge (MCG) defi ned by

$$
\sum_{x, \mu}\left[\operatorname{tr} U_{\mu}^{\Omega}(x)\right]^{2} \stackrel{\Omega}{\longrightarrow} \max
$$




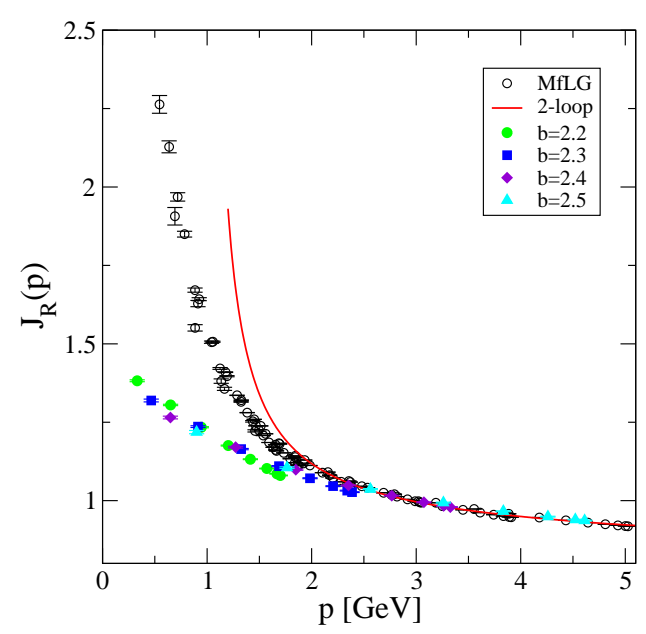

Figure 1: The ghost form factor (full symbols) in MCG as function of momentum transfer. The ghost form factor in Minimal Landau gauge (open symbols, data from [8]). Two loop perturbation theory (solid line).

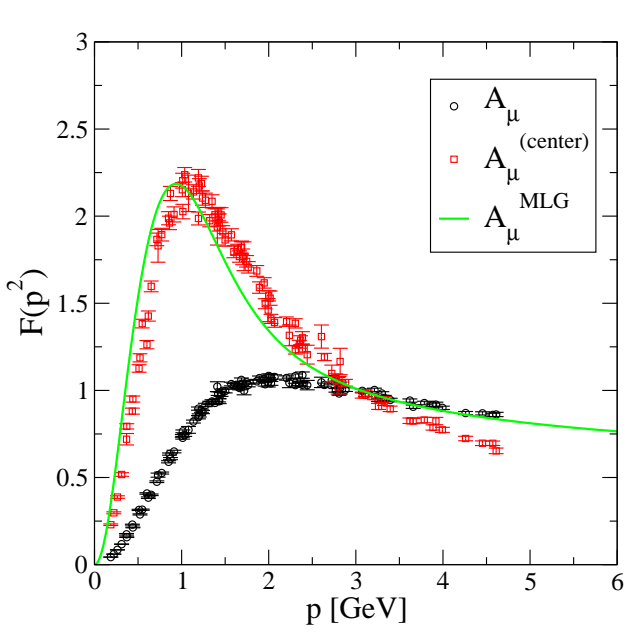

Figure 2: The gluon propagator $F\left(p^{2}\right) / p^{2}$ in MAdL gauge as function of the momentum transfer $p$. The adjoint gluon form factor the gluon fields in MAdL gauge (circles), the center field form factor (squares) and the fit to the gluon form factor in Minimal Landau gauge (line).

This gauge fi xes the gauge group only up to center gauge transformations, i.e. it fixes only the coset $S U(2) / Z(2)=S O(3)$ and brings a given link $U_{\mu}(x)$ as close as possible to a center element $( \pm 1$ for $S U(2)$ ). This gauge is just the minimal Landau gauge for the adjoint representation which does not feel the center. Once, this gauge is implemented, center projection implies to replace a link $U_{\mu}(x)$ by its closest center element, which is given by

$$
Z_{\mu}(x)=\operatorname{sign} \operatorname{tr} U_{\mu}^{\Omega}(x) .
$$

The center projected confi gurations $Z_{\mu}(x)$ form 3-dimensional volumes of links $Z_{\mu}(x)=-1$, the closed boundaries of which represent the center vortices. We separate the center projected vortices from the original gauge fi elds, by writing [7]

$$
U_{\mu}^{\Omega}(x)=Z_{\mu}(x) \bar{U}_{\mu}(x) .
$$

Fig. 2 shows the ghost form factor of the MCG obtained on $16^{4}$ and $24^{4}$ lattices and for $\beta$ values of the Wilson action in the range $\beta \in[2.15,25]$. At high momenta the ghost form factor of MCG approaches the one of the minimal Landau gauge but differs drastically from the latter in the infrared: While the form factor of the minimal Landau gauge is infrared divergent (what is considered as a signal of confi nement), the one of MCG, which does not feel the center, seems to be infrared fi nite. This is consistent with the result obtained in [5], that the infrared divergent behaviour of the ghost form factor in minimal Landau gauge disppears, when center vortices are removed from the Yang-Mills ensemble, i.e. when the full links $U_{\mu}^{\Omega}(x)$ (4) are replaced by their coset parts $\bar{U}_{\mu}(x)$.

The MCG condition (2) does not feel the center and accordingly the Faddeev-Popov operator (and thus the ghost form factor) of MCG depends only on the coset fi elds, $\bar{U}_{\mu}(x)$, and consequently does 


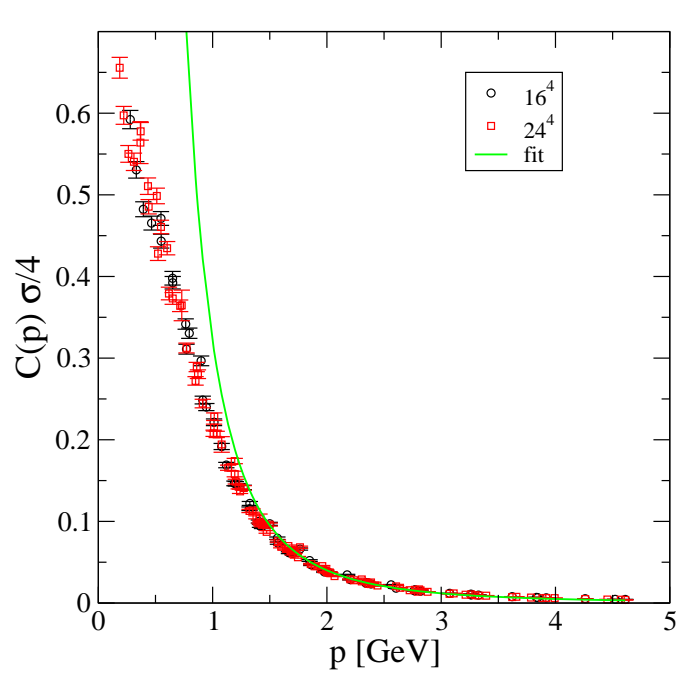

Figure 3: Center field correlation function as func- Figure 4: Center field correlation function as function of momentum for two different lattice sizes.

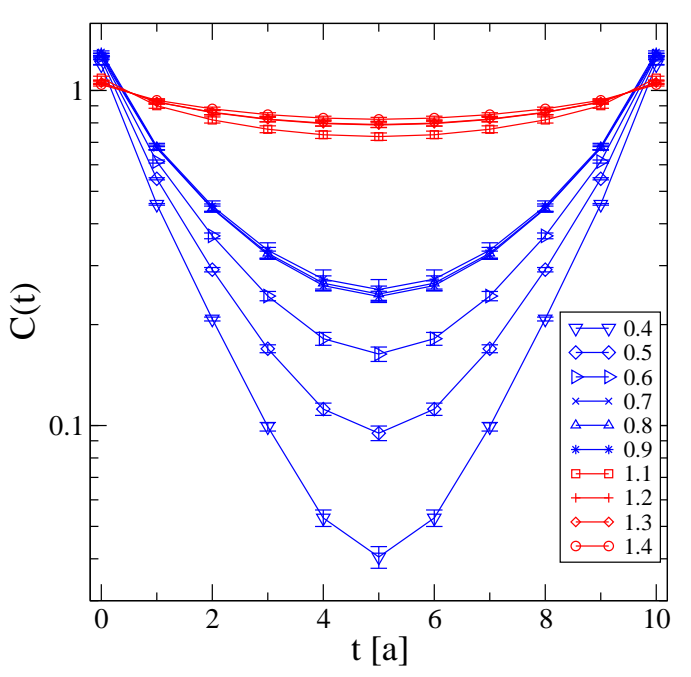

tion of Euclidean time for several temperatures (in units of the critical temperature).

not change when center vortices are removed. Indeed, with respect to the gauge fi xing functional of the MCG (2) the center vortices $\left\{Z_{\mu}(x)=-1\right\}$ are degenerate with the vacuum $\left\{U_{\mu}(x)=1\right\}$ and for a pure center vortex confi guration $\left\{\bar{U}_{\mu}(x) \equiv 1\right\}$ the Faddeev-Popov operator of the MCG becomes the ordinary Laplacian $(-\Delta)$. Hence the center vortices are not on the Gribov horizon of the MCG, as opposed to that of the minimal Landau gauge.

Fig. 1 shows the form factor of the propagator of the gauge fi eld extracted in the standard fashion from the coset fi eld confi gurations $\bar{U}_{\mu}(x)$. For large momenta this quantity reproduces the perturbative result for the full gluon form factor in Landau gauge. Like the full gluon form factor in Landau gauge, $F_{\text {ad }}\left(p^{2}\right)$ vanishes for $p \rightarrow 0$, signaling a mass gap in the excitation spectrum of $\bar{A}_{\mu}(x)$. Finally, $F_{\text {ad }}\left(p^{2}\right)$ deviates essentially from the gluon form factor of minimal Landau gauge in the intermediate momentum regime.

Consider now the center projected confi gurations $\left\{Z_{\mu}(x)\right\}$ (3). Each link $Z_{\mu}(x)$ defi nes an elementary cube on the dual lattice and the total number of non-trivial center links $Z_{\mu}(x)=-1$ defi nes open hypersurfaces $\Sigma$ bounded by closed center vortex surfaces $\partial \Sigma$.

Introducing the characteristic function of the hypersurfaces $\Sigma$ by

$$
a \chi_{\mu}(\Sigma, x)=\frac{1}{2}\left(1-Z_{\mu}(x)\right)=\left\{\begin{array}{l}
1, x \in \Sigma \\
0, x \notin \Sigma
\end{array},\right.
$$

one can defi ne a vector fi eld

$$
\mathscr{A}_{\mu}(\Sigma, x)=\pi \tau_{3} \chi_{\mu}(\Sigma, x)
$$

satisfying $\exp \left\{\operatorname{iaA}_{\mu}(x)\right\}=Z_{\mu}(x)$.

We also use the analogue of the Landau gauge for the center projected fi elds

$$
\sum_{x, \mu} Z_{\mu}(x) \stackrel{\Omega_{2}}{\longrightarrow} \max
$$


where $\Omega_{2}$ is a $Z(2)$ gauge transformations. This gauge condition, eq. (7), minimizes the number of $Z_{\mu}(x)=-1$ links and thus minimizes the volume of the open 3-dimensional hypersurfaces $\Sigma$. Since, the minimal open hypersurfaces are completely determined by their boundary ${ }^{1}$, it is expected that they scale properly towards the continuum limit, if the vortex surfaces $\partial \Sigma$ do. The probability $\mathscr{P}$ that a given link element $Z_{\mu}(x)$ is negative should scale in the continuum limit $a \rightarrow 0$ as $\mathscr{P}=\kappa a$, with $\kappa$ being independent of $a$. This is indeed confi rmed by lattice calculations [9]. Our numerical calculations yield $\kappa \approx 0.26(1) \sqrt{\sigma}$.

Consider now the connected center fi eld correlation function

$$
\begin{array}{r}
a^{2} c_{\mu v}(x-y)=\left\langle Z_{\mu}(x) Z_{v}(y)\right\rangle-\left\langle Z_{\mu}(x)\right\rangle\left\langle Z_{v}(y)\right\rangle . \\
\quad=a^{2} \frac{4}{\pi^{2}}\left[\left\langle\mathscr{A}_{\mu}(x) \mathscr{A}_{v}(x)\right\rangle-\left\langle\mathscr{A}_{\mu}(x)\right\rangle\left\langle\mathscr{A}_{v}(x)\right\rangle\right]
\end{array}
$$

The factor $a^{2}$ was introdcued for later convenience. Although $Z_{\mu}(x)$ is an integer valued fi eld, the propagator $c_{\mu v}(x-y)$, emerging from an ensemble average, appears to be smooth and transverse: $\partial_{\mu} c_{\mu v}(x-y)=0$.

One of our important fi ndings is that the center fi eld correlation function $C_{\mu v}(p)$ is independent of the lattice spacing. The consequences are two-fold: Firstly, the propagator $C_{\mu \nu}(p)$ is not subjected to wave function renormalization. Secondly, $C_{\mu v}(p)$ behaves as a genuine gluonic correlation function with mass dimension two.

Our numerical results for the propagator $C_{\mu \nu}$ in momentum space are shown in fi gure 3. Most striking is that the high momentum tail is well fi tted by the power law (see fi gure 3 )

$$
C(p) / 4=\frac{3.7(1) \sqrt{\sigma}}{p^{3}}, \quad p \geq 2 \mathrm{GeV} .
$$

This implies that the center fi eld correlator is sub-leading in the high momentum regime compared with the perturbative correlator (see also fi gure 2). The center fi eld correlator, however, contributes to the operator product corrections. Hence, the center "background" fi eld could serve a natural explanation for the condensates entering the operator product expansion. Finally note that $F^{\text {cen }}\left(p^{2}\right)$ is enhanced in the low momentum regime, where it has the same shape as the gluon form factor in minimal Landau gauge.

Let us fi nally consider the deconfi nement phase transition at high temperatures. This transition is well understood in the vortex picture where it appears as vortex depercolation transition. Since the transition is of 2 nd order, it is accompanied by the occurrence of a massless excitation. However, it is known that neither the gluonic mass gap nor the color singlet states hardly change signifi cantly and thus cannot be identifi ed with the excitation that becomes massless at the transition. Because of the success of the vortex picture in describing this transition, one might suspect that the center fi eld correlator contains the desired information. We have therefore studied the correlator $\sum_{\vec{x}} c(t, \vec{x})$ as function of $t$ for several temperatures. Simulations have been carried out using

\footnotetext{
${ }^{1}$ : For topologically non-trivial surfaces $\partial \Sigma$ there may be more than one (relative) minimal open surfaces $\Sigma$. Nevertheless all these minimal surfaces are completely determined by their common boundary $\partial \Sigma$.
} 
a $30^{3} \times 10$ lattice. $\beta$ was varied from 1.91 to 2.69 to adjust the temperature. The result is shown in fig. 4. At temperatures $T$ below the critical temperature $\left(T_{c}\right)$ we fi nd an exponential decrease of the correlator $C(t)$, while the correlation is compatible with a power-law for $T>T_{c}$.

\section{References}

[1] J. Greensite, "The confinement problem in lattice gauge theory," Prog. Part. Nucl. Phys. 51, 1 (2003) [arXiv:hep-lat/0301023].

[2] V. N. Gribov, “Quantization Of Non-Abelian Gauge Theories,” Nucl. Phys. B 139, 1 (1978).

[3] D. Zwanziger, “Critical limit of lattice gauge theory,” Nucl. Phys. B 378, 525 (1992).

[4] R. Alkofer and L. von Smekal, "The infrared behavior of QCD Green's functions: Confinement, dynamical symmetry breaking, and hadrons as relativistic bound states," Phys. Rept. 353, 281 (2001) [arXiv:hep-ph/0007355].

[5] J. Gattnar, K. Langfeld and H. Reinhardt, "Signals of confinement in Green functions of SU(2) Yang-Mills theory,” Phys. Rev. Lett. 93, 061601 (2004) [arXiv:hep-lat/0403011].

[6] J. Greensite, S. Olejnik and D. Zwanziger, "Center vortices and the Gribov horizon," JHEP $\mathbf{0 5 0 5}$, 070 (2005) [arXiv:hep-lat/0407032].

[7] P. de Forcrand and M. D'Elia, “On the relevance of center vortices to QCD," Phys. Rev. Lett. 82, 4582 (1999) [arXiv:hep-lat/9901020].

[8] J. C. R. Bloch, A. Cucchieri, K. Langfeld and T. Mendes, "Propagators and running coupling from SU(2) lattice gauge theory,” Nucl. Phys. B 687, 76 (2004) [arXiv:hep-lat/0312036].

[9] A. V. Kovalenko, M. I. Polikarpov, S. N. Syritsyn and V. I. Zakharov, "Three dimensional vacuum domains in four dimensional SU(2) gluodynamics," arXiv:hep-lat/0408014. 